This item was submitted to Loughborough's Research Repository by the author.

Items in Figshare are protected by copyright, with all rights reserved, unless otherwise indicated.

\title{
A study of decodable breathing patterns for augmentative and alternative communication
}

PLEASE CITE THE PUBLISHED VERSION

https://doi.org/10.1016/j.bspc.2020.102303

PUBLISHER

Elsevier BV

VERSION

AM (Accepted Manuscript)

\section{PUBLISHER STATEMENT}

This paper was accepted for publication in the journal Biomedical Signal Processing and Control and the definitive published version is available at https://doi.org/10.1016/j.bspc.2020.102303

\section{LICENCE}

CC BY-NC-ND 4.0

\section{REPOSITORY RECORD}

Elsahar, Yasmin, Sijung Hu, Kaddour Bouazza-Marouf, David Kerr, Will Wade, Paul Hewett, Atul Gaur, and Vipul Kaushik. 2020. "A Study of Decodable Breathing Patterns for Augmentative and Alternative Communication”. Loughborough University. https://hdl.handle.net/2134/13295870.v1. 


\title{
Highlights
}

\section{A Study of Decodable Breathing Patterns for Augmentative and Alternative Communication}

\author{
Yasmin Elsahar ${ }^{1}$, Sijung Hu ${ }^{1 *}$, Kaddour Bouazza-Marouf ${ }^{1}$, David Kerr ${ }^{1}$, Will Wade ${ }^{2}$, Paul \\ Hewett $^{2}$, Atul Gaur ${ }^{3}$, and Vipul Kaushik ${ }^{3}$ \\ ${ }^{1}$ Wolfson School of Mechanical, Electrical, and Manufacturing Engineering, Loughborough \\ University, Loughborough, LE11 3TU, UK \\ ${ }^{2}$ ACE Centre, Hollinwood Business Centre, Albert Street, Oldham, OL8 3QL, UK \\ ${ }^{3}$ University Hospitals of Leicester NHS Trust, Leicester, LE3 9QP, UK \\ *Correspondence: S.Hu@lboro.ac.uk
}

\section{Highlights}

- Breathing patterns to be used for augmentative and alternative communication (AAC) purposes.

- An optimized dynamic air pressure detection system to capture modulated breathing patterns for AAC.

- Elastic measures for a superior performance to classify modulated breathing patterns.

- Tradeoffs to be established to balance the speed of spoken output generation and DTW complexity. 


\title{
Article
}

\section{A Study of Decodable Breathing Patterns for Augmentative and Alternative Communication}

\author{
Yasmin Elsahar ${ }^{1}$, Sijung Hu ${ }^{1 *}$, Kaddour Bouazza-Marouf ${ }^{1}$, David Kerr ${ }^{1}$, Will Wade ${ }^{2}$, Paul \\ Hewett $^{2}$, Atul Gaur ${ }^{3}$, and Vipul Kaushik ${ }^{3}$ \\ ${ }^{1}$ Wolfson School of Mechanical, Electrical, and Manufacturing Engineering, Loughborough \\ University, Loughborough, LE11 3TU, UK \\ ${ }^{2}$ ACE Centre, Hollinwood Business Centre, Albert Street, Oldham, OL8 3QL, UK \\ ${ }^{3}$ University Hospitals of Leicester NHS Trust, Leicester, LE3 9QP, UK \\ *Correspondence: S.Hu@lboro.ac.uk
}

\begin{abstract}
People who use high-tech augmentative and alternative communication (AAC) solutions still face restrictions in terms of practical utilization of present AAC devices, especially when speech impairment is compounded with motor disabilities. This study aims to explore an effective way to decode breathing patterns for AAC by the means of a breath activated dynamic air pressure detection system (DAPDS) and supervised machine learning (ML). The aim is to detect a user's modulated breathing patterns (MBPs) and turn them into synthesized messages for conversation with the outside world. MBPs are processed using a one-nearest neighbor (1-NN) algorithm with variations of dynamic time warping (DTW) to produce synthesized machine spoken words (SMSW) at managed complexities and speeds. An ethical approved protocol was conducted with the participation of 25 healthy subjects to create a library of 1500 MBPs corresponding to four different classes. A mean systematic classification accuracy of $91.97 \%$ was obtained using the current configuration. The implications from the study indicate that an improved AAC solution and speaking biometrics decoding could be undertaken in the future.
\end{abstract}

Keywords: Piecewise dynamic time warping (PDTW); breathing patterns; augmentative and alternative communication (AAC); dynamic air pressure detection system (DAPDS); supervised machine learning; $k$-nearest neighbor; synthesized machine spoken words 


\section{List of Abbreviations}

\begin{tabular}{l|l} 
AAC & $\begin{array}{l}\text { Augmentative and Alternative Communication } \\
\text { DAPDS }\end{array}$ \\
DTwamic Air Pressure Detection System \\
$k$-NN & $\begin{array}{l}\text { Dynamic Time Warping } \\
\text {-Nearest Neighbours }\end{array}$ \\
ML & Machine Learning \\
MBPI & Modulated Breathing Patterns Interpretation \\
MBP(s) & Modulated Breathing Pattern(s) \\
PCB & Printed Circuit Board \\
PDTW & Piecewise Dynamic Time Warping \\
TP & True Positive
\end{tabular}

\section{Introduction}

Augmentative and alternative communication (AAC) methods have been widely developing over the recent years, aiding individuals who have lost their speech abilities to reconnect with the outside world and obtain a form of free expression. Low-tech AAC methods predominantly rely on communication books and board displays, whereas high-tech AAC methods utilize specialized software and hardware applications to fulfil a user's communication target. High-tech AAC has been undergoing substantial research over the last 30 years [1]. It primarily involves accessing a specialized AAC platform through a variety of activation methods, including eye gaze [2,3], brain-computer interface [4], respiratory signals [5], switch access [3,6], or touch-based applications [7]. The widespread of smart devices has been aiding the ability of specialized and non-specialized AAC applications to become widely available [8,9]. High-tech AAC has also started to provide dynamic solutions for communication, including personal narrative systems for social interaction, which are usually interconnected with Natural Language Processing [10,11]. Nonetheless, research under the umbrella of recent AAC developments confirms that a wider scale of technology utilization is needed to fulfil the needs of AAC users [12,13]. High-tech AAC solutions are considered generally expensive [7], complex, and usually require significant efforts from the user in terms of training, rendering many solutions cumbersome. Studies [2,5] also show that the extended lexicons of phrases and sentences preprogramed into a device confine the flexibility of conversing in unique messages. Moreover, AAC devices dependent on motor movements for access could be restrictive for users who suffer motor impairments [14]. Therefore, evolving AAC interventions need to consider a set of factors, primarily focusing on the complexity, affordability, and ease of access to the solution. User preferences and the speeds at which the alternative signals are acquired, especially for users with associated developmental disabilities, also need to be considered $[15,16]$. 
In this study, an alternative AAC solution, based on encoding of continuous modulated breathing patterns (MBPs), is being researched to address a shortfall in this area. Breath activated solutions require negligible motor movements from the users to encode messages. Early studies in this field were initiated in [17], where an elastic thoracic belt activated via respiratory diaphragm movements is used to move a mouse across a computer screen to select letters from a predictive language software and spell words via fine breath tuning. Studies $[12,18,19]$ also reported the usage of digitized respiratory signals for the purpose of communication. For example, sniffing signals were utilized to spell letters to form words and were also used as a switch access method to move a wheelchair for quadriplegic patients. It was also shown in [19] that variations of breathing blows encoded to produce specific Morse Code signals to spell letters could be used for AAC purposes.

Although the listed approaches in [12,17-19] were beneficial in allowing free user expression, digitally encoded breathing signals are cumbersome to generate, as the users go through a learning curve to breathe in a specific way to create a sequence of dots and dashes representing the intended letters or phrases. Encoding breathing signals in an analogue format, which preserves their amplitude, phase, and/or frequency content is more natural to generate, and provides a richer information content that may be utilized for communication. For example, two identical signals differing in amplitude could represent nuance and grammatical deviations. In the context of analogue breath encoding, our previous study presented in [5] utilized a unidirectional microphone to fulfil this AAC target via encoded analogue breathing signals. Analogue encoding of MBPs was found as a promising approach for AAC purposes. However, the work in [5] concluded that the usage of a microphone/acoustic transducer to detect sets of MBPs restricted the information content of the signal, specifically the inhalation profiles of the MBPs, as they exerted a very low pressure on the diaphragm of the microphone. An improved representation is therefore necessary to ensure the user-selected patterns are accurately represented. Moreover, a need to speed up the signal processing and associated computational demands was demonstrated to aid in the acceleration of the MBP recognition process, allowing users to communicate at a faster rate.

The purpose of this study is to explore an effective way to decode breathing patterns for AAC. To reach this, an improved and validated AAC breath activated system was established by integrating a dynamic air pressure detection system (DAPDS) to comprehensively record modulated breathing profiles. A supervised machine learning (ML) algorithm was introduced to recognize MBPs and robustly produce synthesized machine spoken words (SMSW). The intended solution considers an accuracy rate of at least $90 \%$ to allow for reliable, real-time user expression.

\section{Materials and Methods}

\subsection{Modulated Breathing Patterns Interpretation Platform}

Individuals with speech disabilities must have the ability to spontaneously control their breathing rates in order to use the modulated breathing patterns interpretation (MBPI) 
platform. The designed platform encompasses two modes of operation, as demonstrated in Figure 1:

- Mode 1: The training mode (also known as the learning mode), where the user teaches the system new phrases by providing MBP repetitions, creating an extended library of phrases/sentences linked to each user generated MBP. Each unique phrase/sentence is assigned to a "class" handler. The user is recommended to generate at least ten MBP repetitions for each intended phrase/sentence. These multiple variations of the same MBP would be beneficial to train the ML algorithm and optimize the MBPs' recognition accuracy. The appropriate method of selecting the phrases/sentences would depend on the type of speech disability.

- Mode 2: The live mode, where the system recognizes and classifies a user's incoming MBP to produce a synthesized machine spoken output or pre-recorded speech stored in the subject's own voice (for users who have deteriorating speech conditions).

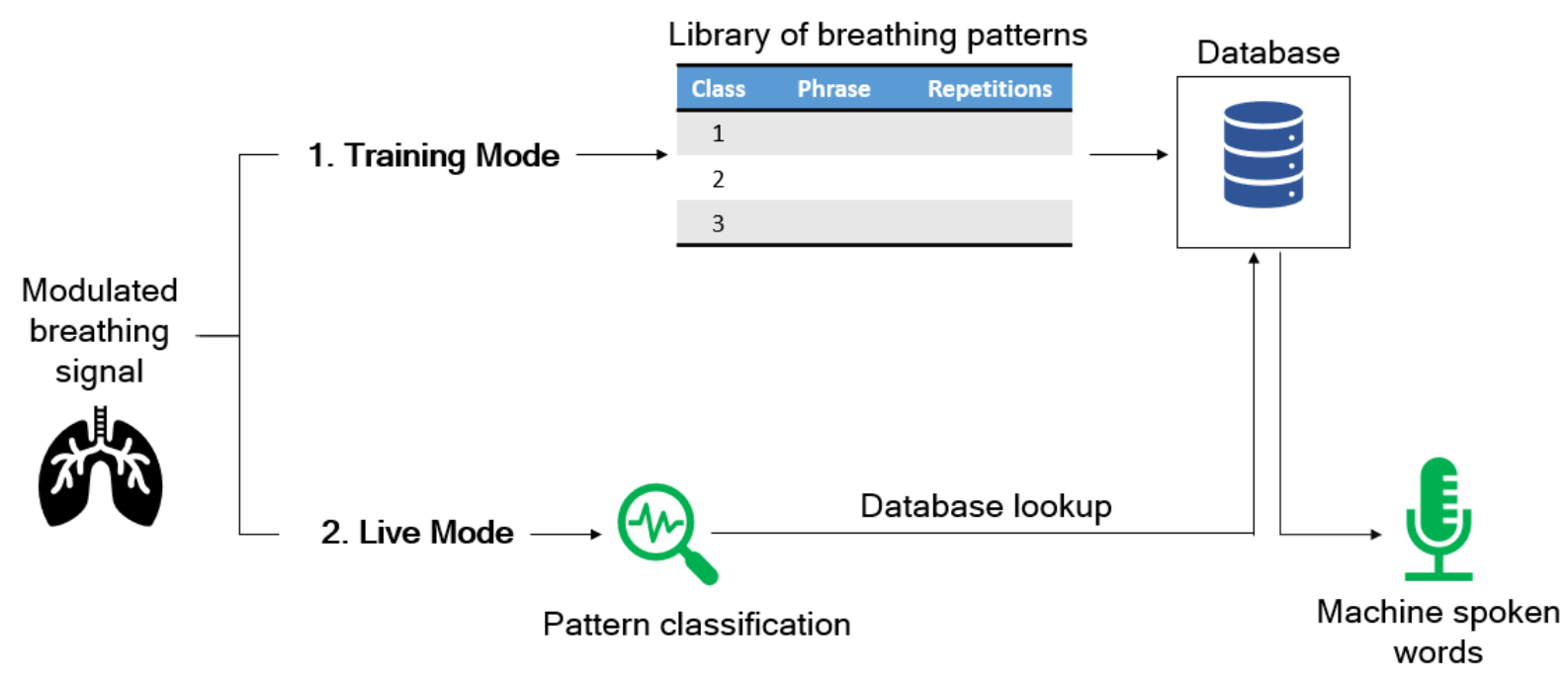

Figure 1 Schematic architecture of the modulated breathing patterns interpretation (MBPI) platform, including the supported operational modes, in line with the typical breath encoding architecture in [5,12].

\subsection{Dynamic Air Pressure Detection System (DAPDS)}

A dynamic air pressure detection system (DAPDS) to capture and process MBPs has been developed at Loughborough University. Figure 2 shows the current hardware prototype of the DAPDS developed for this study. It consists of a headband unit to which a digital, low differential pressure sensor (type: LMIS500BB3S, pressure range: $\pm 500 \mathrm{~Pa}$, supplied by First Sensor) is connected to a Bluno Nano microcontroller board via a printed circuit board (PCB) of dimensions $17.6 \times 11.2 \mathrm{~mm}$. $\mathrm{I}^{2} \mathrm{C}$ communication lines are utilized between the master microcontroller board and the slave pressure sensor to synchronize and read the data. A 20 $\mathrm{cm}$ long air tube together with a parabolic diffuser cap (at its distal end) are used to collect and transfer the pressure signals to the pressure sensor. A flexible copper wire is externally attached to the air tube to provide a stable means of orienting the diffuser cap of the headset towards the user's mouth. 


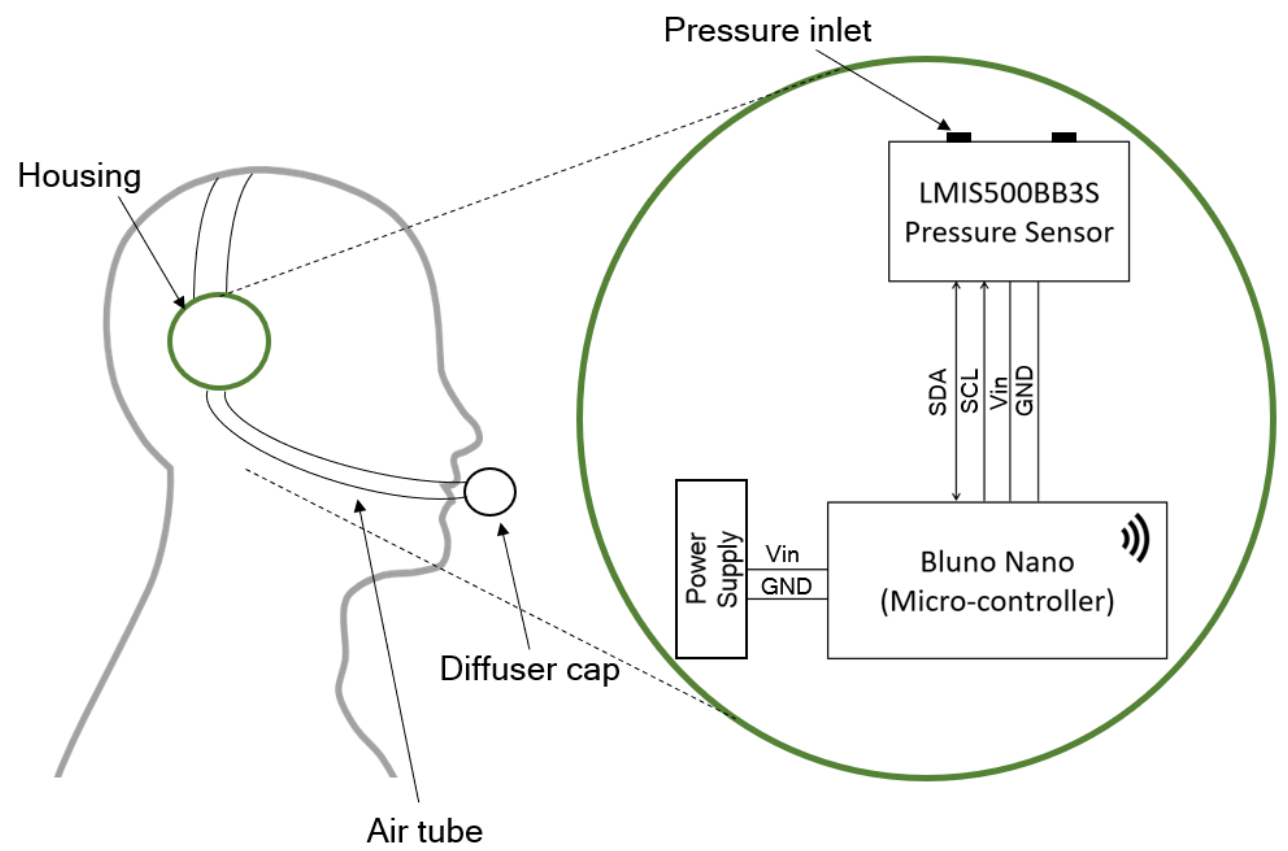

Figure 2 The Dynamic Air Pressure Detection System (DAPDS) hardware, displaying an air tube attached to the headset arm and a unit housing the microcontroller board, thermal pressure sensor and a power supply.

\subsection{Modulated Breathing Signals Transfer and Data Logging}

An Arduino sketch was uploaded to the Bluno Nano board to initiate the $\mathrm{I}^{2} \mathrm{C}$ serial connection between the LMI sensor and the Bluno board and looped to read the detected breathing pressure values. The sensor was calibrated in accordance with the manufacturer's datasheet. The overall DAPDS prototype together with a sample MBP obtained using this hardware configuration are shown in Figure 3. To record the MBPs on a PC, an add-on text logger for microcontroller data acquisition on Excel (PLX) was used. The maximum data rate using this configuration in a wired format was recorded at 128 samples/second.

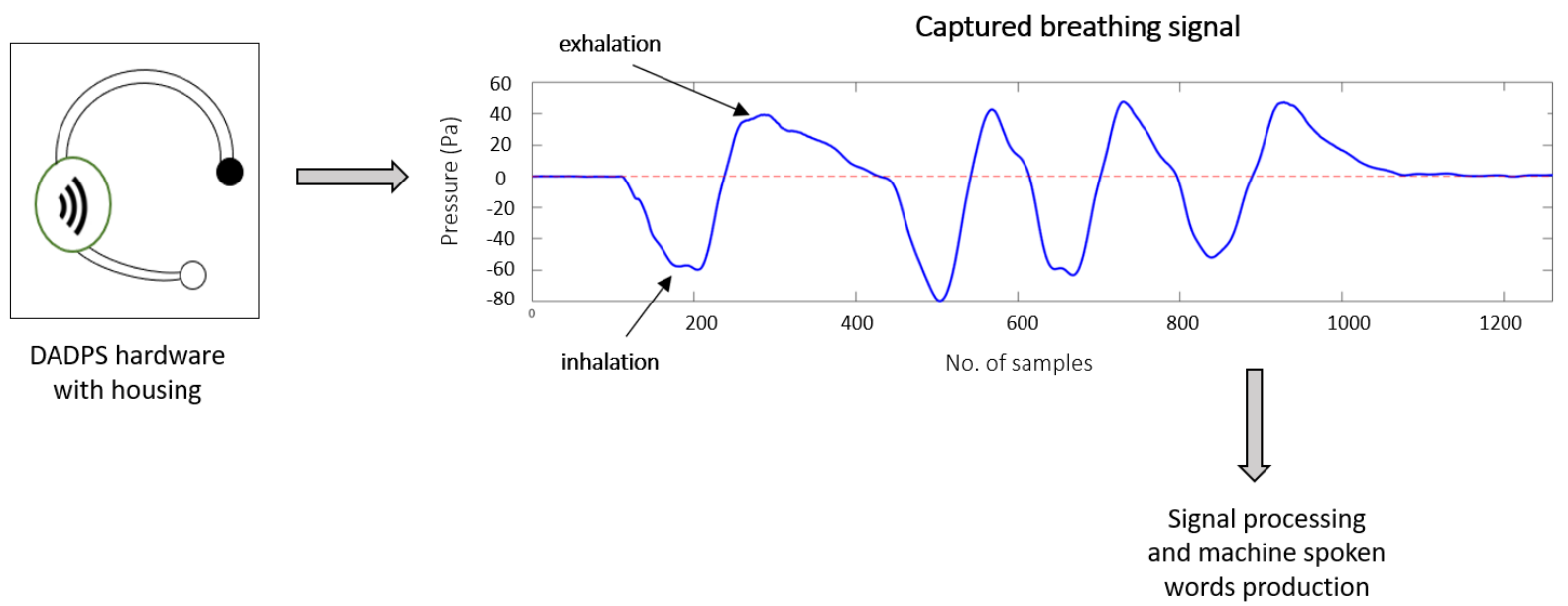

Figure 3 Overview of the MBPI platform and the DAPDS hardware, including the comprehensive profile of a typical sample of a captured MBP. 


\subsection{Experimental Protocol}

An experimental protocol to collect MBPs from 25 healthy, non-speech disabled volunteers (age: 18-50) was approved by Loughborough University's Ethics Committee. All subjects completed an informed consent form prior to taking part in the study. As detailed in Figure 4, and in line with the protocol in [5], each subject was first asked to don the developed DAPDS hardware and position the arm with the diffuser cap close to the mouth. A set of trial MBPs were recorded to familiarize the subject with the process of breathing modulation. The two modes of operation were next tested:

- Training mode: Each subject was asked to modulate four MBPs of their own selection, with each of these four MBPs representing a user-selected phrase/sentence. Ten repetitions of each MBP were acquired to build up the library of training MBPs (total: 40 training MBPs/subject). Each set of ten MBPs representing the same phrase was assigned to a unique class handler to label the MBPs in preparation of the supervised ML recognition.

- Live mode: The investigator re-asks the participant to don the headset unit. The subject provides five more repetitions of each of the four selected MBPs (total: 20 live MBPs/subject). This is carried out in an attempt to recognize the triggered MBP and identify the associated training MBP class and the words/phrases associated with this pattern.

Upon the completion of the acquisition, the session was concluded. A sample of a subject's acquired MBPs is shown in the waterfall plots of Figure 5, where the four MBPs are displayed along with their training and live repetitions. A total of 1000 training MBPs and 500 live MBPs were acquired from the 25 subjects during this protocol.

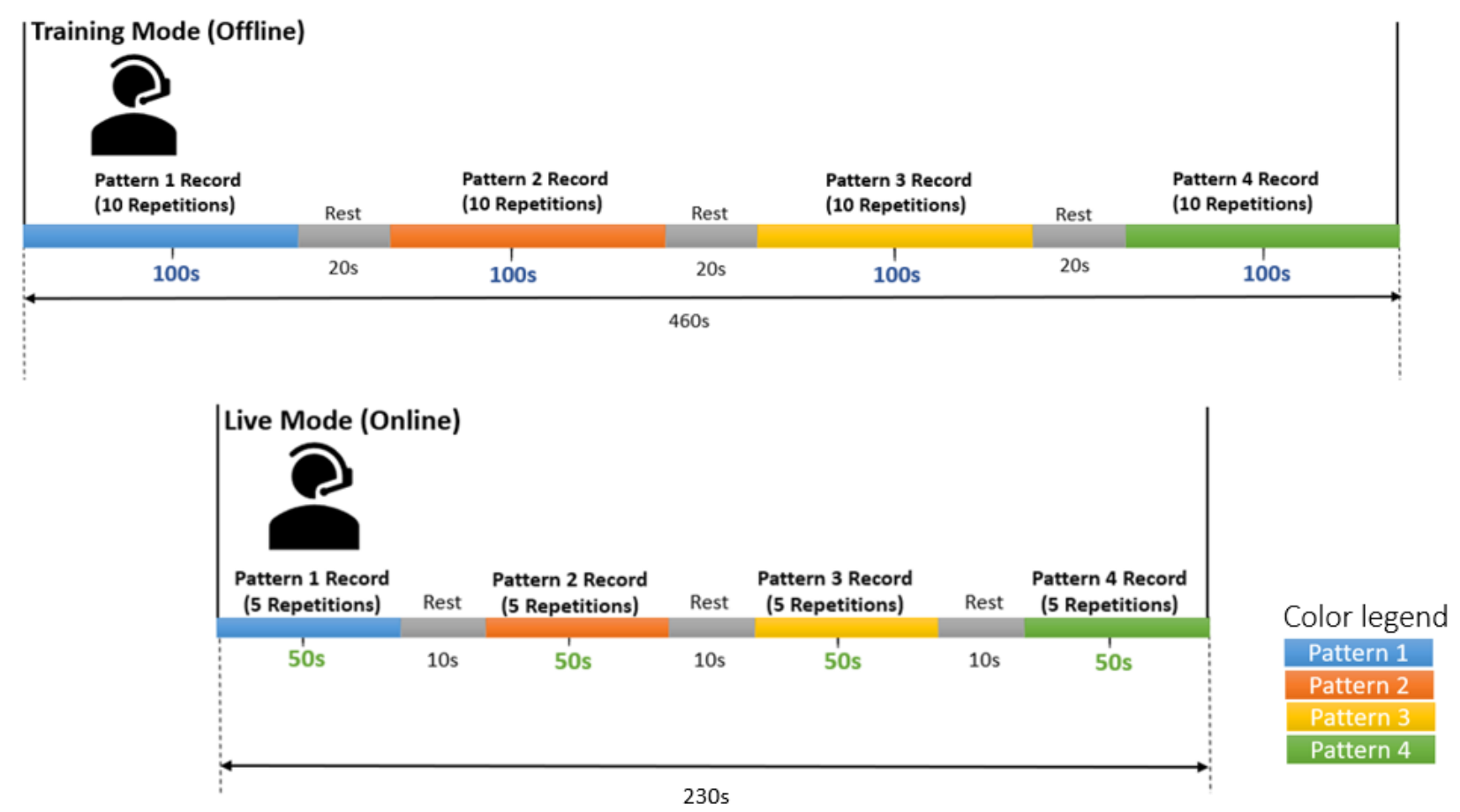

Figure 4 Protocol structure, including the time windows of data acquisition and MBP recording for the training and live modes, in line with [5]. 


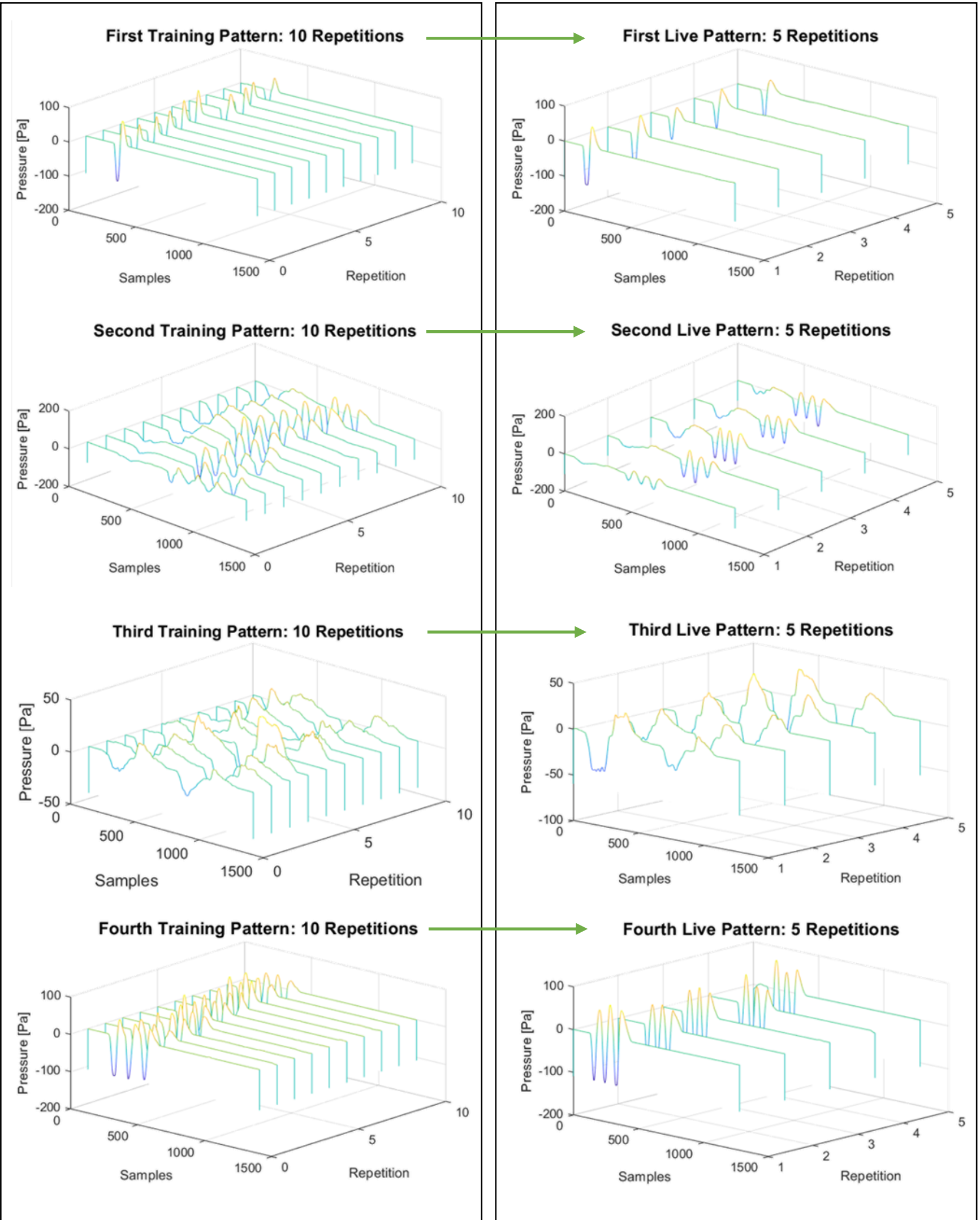

Figure 5 A typical sample of waterfall plots of a subject's MBP recordings, including 40 training MBPs belonging to four different classes, and their associated 20 live MBPs. 


\subsection{Supervised Machine Learning for MBPs Recognition}

For the generation of a correct spoken output, the live MBPs produced by the user must be appropriately matched to the training MBPs corresponding to the intended phrase/sentence. Several considerations regarding the user-generated data are investigated prior to the selection of a classification algorithm for testing. The user-generated MBPs are temporal sequences recorded over short time windows and are assumed to be time series over the specified windows. However, as the patterns are human-generated, they are susceptible to basic variations (as shown in Figure 6), including:

- Time shift: temporal variation between the MBPs, and

- Spectral variation: narrowing and widening of the detected pressure peaks and troughs representative of the inhalation and exhalation.

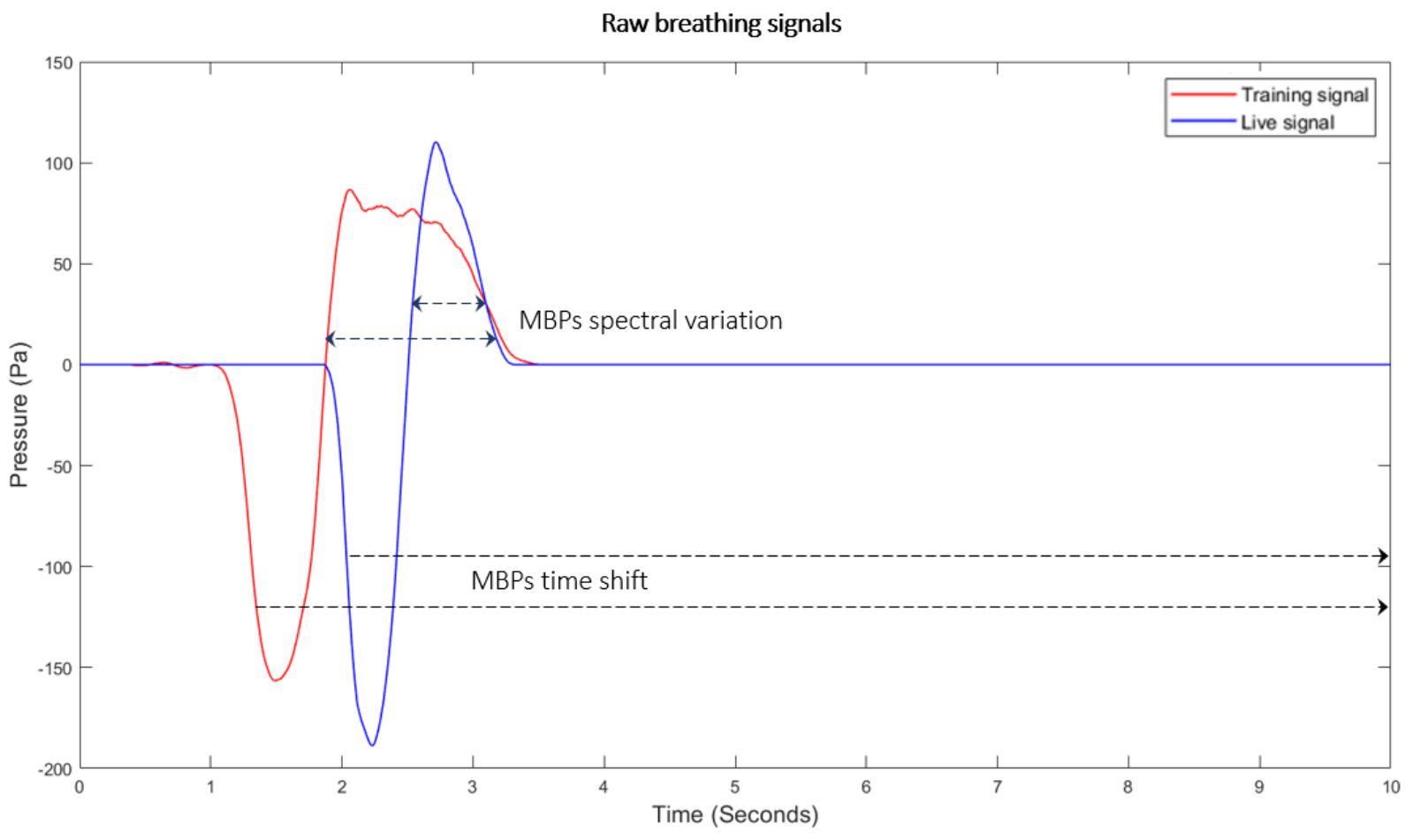

Figure 6 MBPs structural variations (sample of a random training MBP and a random corresponding live $M B P)$.

The highlighted differences between the structural compositions of any two MBPs (interchangeably and within the same set) constraint the usability of a Euclidean, point-bypoint, matching of the incoming signals. The studies in the applications of dynamic programming demonstrate that a warped, dynamic time warping (DTW) elastic distance in combination with a one-nearest neighbor classifier (1-NN) for the comparison of temporal series has been widely validated for audio, video and data alignments $[13,17-21]$. DTW is predominantly used in conjunction with a $k$-nearest neighbor $(k$-NN) classifier, a nonparametric classification technique $[13,22]$. With $k=1$, the classifier associates an input sample with the class of its closest DTW matched neighbor. 


\subsubsection{Data Structures of Training and Live MBPs}

Since MBPs are considered time series, 1-NN DTW has been identified for testing. The training MBPs are recorded over a window of 10 seconds at a sample frequency $\left(f_{s}\right)=128$ samples/second. Ten repetitions of each MBP are provided to train the recognition algorithm $(t=10)$. The training library $(T)$ of patterns is composed of a concatenation of all training series $\left(T x_{t, i}, \ldots, T x_{t, p}\right)$, where $x$ is the index of a distinct class, $t$ is the index referring to the $t^{\text {th }}$ repetition of a pattern of class $T x, i$ is the $i^{\text {th }}$ element of a training series $T x_{t}$, and $p$ is the length of the series. A label is appended with every training series to represent the class to which a series belongs. The structure of the training library is hence given by

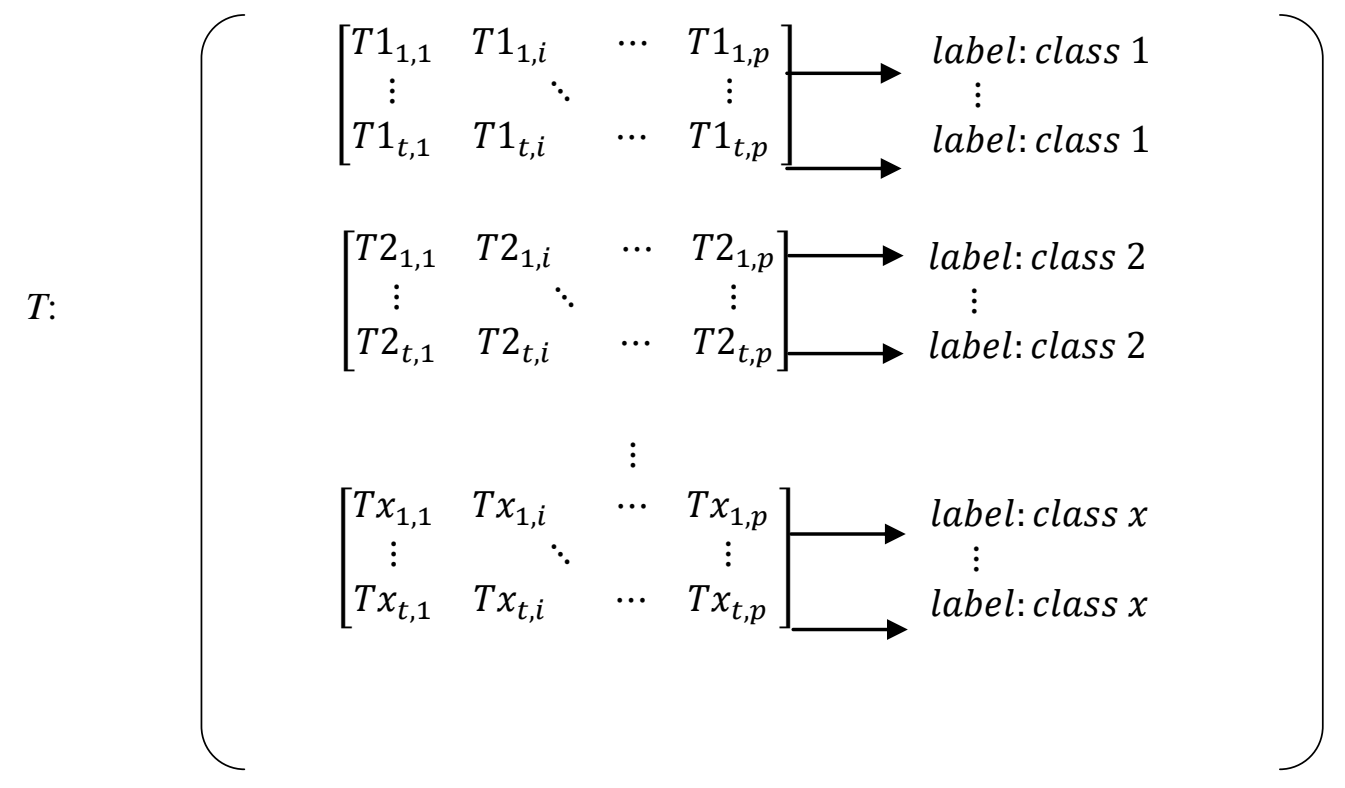

The live MBP inputted by the user is denoted by $L$, such that

$$
L=l_{1}, l_{2}, \ldots, l_{j}, \ldots l_{q}
$$

where $j$ is the $j^{\text {th }}$ element of the live series $L$, and $q$ is the length of the series.

\subsubsection{Piecewise DTW (PDTW) for MBPs Processing}

Computing the distance between a live MBP $(L)$ and the training MBP sequences is based on finding the DTW distance between $L$ and every training series $\left(T x_{t}\right)$ of the training library. The cumulative distance path with the least cost between the live series $L$ and one training series $T x_{t}$ via classical DTW is computed via 


$$
D(i, j)=d\left(T x_{t, i}, l_{j}\right)+\min \left\{\begin{array}{c}
D(i, j-1) \\
D(i-1, j) \\
D(i-1, j-1)
\end{array}\right.
$$

where $d\left(T x_{t, i}, l_{j}\right)$ is the Euclidean distance between $T x_{t, i}$ and $l_{j}$. The warping path $W=$ $w_{1}, w_{2}, \ldots w_{K}$ is constructed such that $w_{k}=(i, j)_{k}$, where $\mathrm{i}$ and $\mathrm{j}$ are the time indices from the series $T x_{t}$ and $\mathrm{L}$ respectively, and $\max (\mathrm{p}, \mathrm{q}) \leq K<\mathrm{p}+\mathrm{q}-1$. However, classical DTW has a quadratic complexity of $\mathrm{O}\left(N^{2}\right)$, which could become computationally demanding as the library of MBPs expands [5,21]. Hence, given that the MBP sequences used in this study are defined in length (1280 samples/MBP), Piecewise DTW (PDTW) was implemented to test the reduction of the computational complexity of the MBPI system's processing algorithm and speed up DTW's classification time. Assuming the live MBP $L=l_{1}, l_{2}, \ldots, l_{j}, \ldots l_{q}$, and $N$ as the target dimensionality of the intended $\bar{L}$ series, given that $1 \leq N \leq q$, authors in [23] demonstrate that the time series $L$ of length $q$ could be represented in $N$ space, such that $\bar{L}=\overline{l_{1}}, \ldots \overline{l_{m}}, \ldots, \overline{l_{N}}$ with the $m^{\text {th }}$ element of $\bar{L}$ calculated as

$$
\overline{l_{m}}=\frac{N}{q} \sum_{z=\frac{q}{N}(m-1)+1}^{\frac{q}{N} m} l_{z}
$$

Such a representation is known as the Piecewise Aggregate Representation (PAA) of the series. The compression rate $(c)$ is dependent on the data structure of the time series and the recognition approach and is best optimized for each system on a case by case basis. To test the feasibility of applying PDTW to the MBPI platform, four compression rates $(c=2.00$, $4.00,5.00$, and 8.00) are selected for two primary reasons. First, the selection of $N$ as a factor of $q$ allows the computation of "whole" frames, rather than fraction of frames [23]. Second, choosing a very high compression factor under-represents the series, and might in turn negatively affect the accuracy of the algorithm. Moreover, given that breathing frequencies are generally very low (12-20 breaths per minute), constraining the series with the listed compression rates was assumed to be acceptable. The constrained lengths of the MBP series based on $c$ are shown in Table 1 .

Table 1 Lengths of MBP series according to the selected compression rate (c).

\begin{tabular}{ccccc}
\hline Compression rate $(c)$ & 2.00 & 4.00 & 5.00 & 8.00 \\
\hline Length of PAA MBP series & 640.00 & 320.00 & 256.00 & 160.00 \\
\hline
\end{tabular}

Based on these assumptions, the PAAs of the live and training series (for all subjects) were computed for each of the four resolutions, and each time series of the training library $(T)$ was represented by its PAA equivalent. The warped path was found between the PAA of a live MBP and the PAA of each training MBP by using the same matching algorithm as classical DTW (equation 3). 


\subsubsection{Data Flow to Produce a Spoken Output}

By applying PDTW to an incoming live signal, the computed distances are stored in a matrix, $\mathrm{D}$, beginning from the distance to the first training pattern $D\left(T 1_{1}, L\right)$ up to the last training pattern $D\left(T x_{t}, L\right)$. The recognized training series with the minimal distance to the live MBP is outputted, together with its corresponding label, and the live MBP is recognized to belong to this training's MBP class. The words/phrases associated with this class are spoken via synthesized speech. Based on this data logic, a flowchart of the system's live mode to produce a spoken output is given in Figure 7.

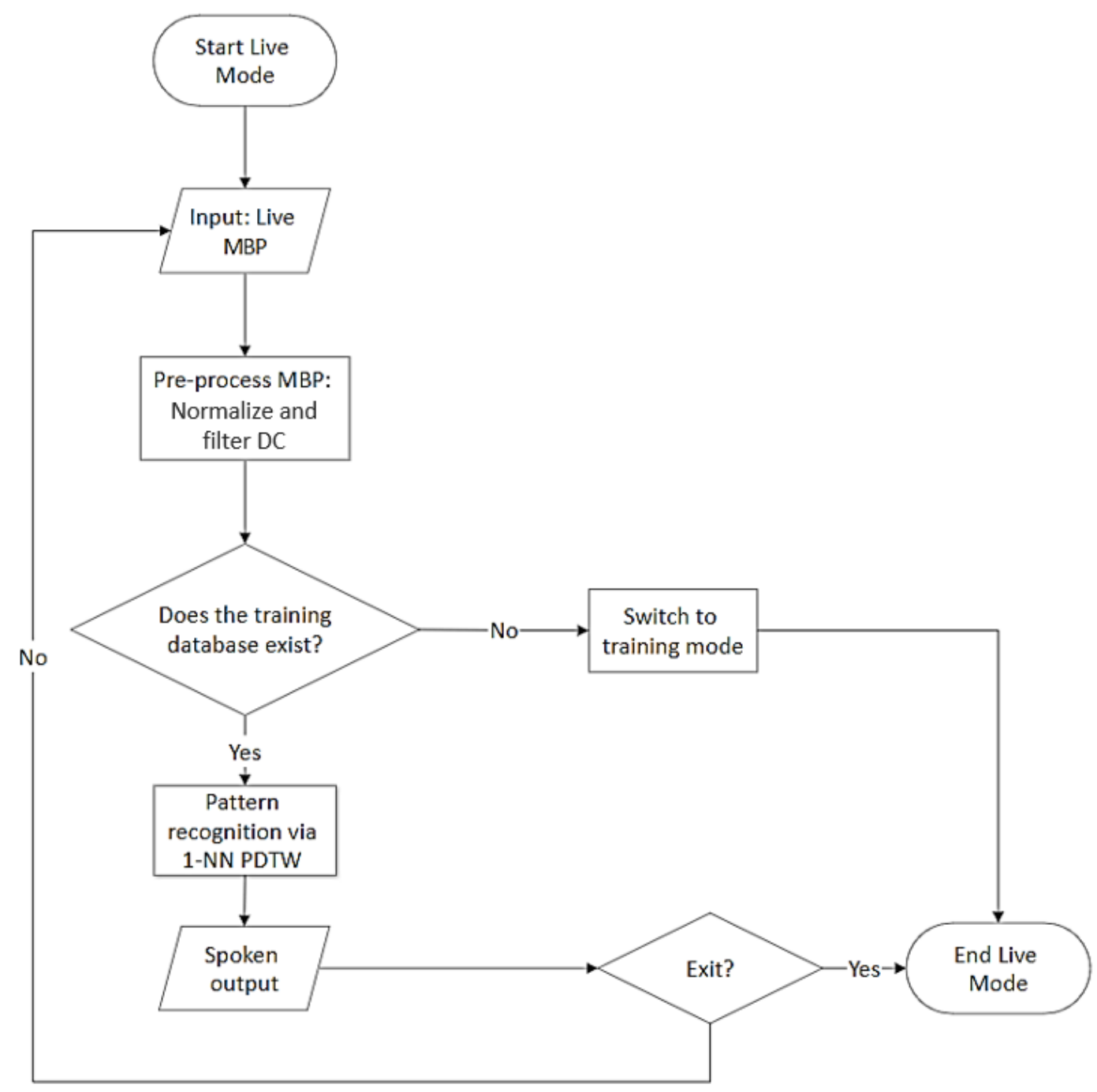

Figure 7 Flowchart of the live mode's MBPs processing via 1-NN PDTW. The process starts with the live MBP modulation and ends with an "exit" prompt for the user to modulate one more live MBP or exit the live mode.

\section{Results}

MBPs were collected and classified using the abstracted PDTW PAA representations of the MBP series presented in Section 2. To obtain the classification accuracy, confusion matrices were generated for each subject's MBPs dataset. Tables 2 and 3 show the aggregated results 
(for 25 subjects) obtained for the lowest and highest compression rates corresponding to the classical DTW $(c=0)$ and PDTW $(c=8.00)$ respectively. The true positive (TP) values were recorded diagonally to represent the matching between a predicted MBP class and an actual MBP class. Each subject's recognized MBP percentage was calculated based on the number of correctly classified MBPs (i.e. TPs) over the total number of live patterns (20 MBPs). The results were aggregated for all 25 subjects and a percentage classification accuracy representative of the overall classification accuracy was recorded. Five sets of live MBPs were excluded due to the subjects' inability to recall and generate the selected MBPs.

Table 2 Confusion matrix results for classical DTW $(c=0):$ No compression, with average accuracy $=91.97 \%$

\begin{tabular}{|c|c|c|c|c|c|}
\hline \multirow{2}{*}{\multicolumn{2}{|c|}{$\begin{array}{c}\text { Classical DTW } \\
(c=0)\end{array}$}} & \multicolumn{4}{|c|}{ Predicted class } \\
\hline & & Class 1 & Class 2 & Class 3 & Class 4 \\
\hline \multirow{4}{*}{$\begin{array}{c}\text { Actual } \\
\text { class }\end{array}$} & Class 1 & $95.24 \%$ & $0 \%$ & $3.81 \%$ & $0.95 \%$ \\
\hline & Class 2 & $1.90 \%$ & $88.60 \%$ & $3.80 \%$ & $5.70 \%$ \\
\hline & Class 3 & $1.90 \%$ & $2.86 \%$ & $87.62 \%$ & $7.62 \%$ \\
\hline & Class 4 & $0 \%$ & $0 \%$ & $3.60 \%$ & $96.40 \%$ \\
\hline
\end{tabular}

Table 3 Confusion matrix results for PDTW (c=8.00): Highest compression rate, with average accuracy = $90.00 \%$

\begin{tabular}{cccccc}
\hline \multirow{2}{*}{ PDTW $(c=8.00)$} & \multicolumn{4}{c}{ Predicted class } \\
\cline { 2 - 6 } & & Class 1 & Class 2 & Class 3 & Class 4 \\
\hline \multirow{3}{*}{$\begin{array}{c}\text { Actual } \\
\text { class }\end{array}$} & Class 1 & $\mathbf{9 4 . 5 5 \%}$ & $3.64 \%$ & $1.81 \%$ & $0 \%$ \\
\cline { 2 - 6 } & Class 2 & $2.86 \%$ & $\mathbf{8 7 . 6 2 \%}$ & $0.95 \%$ & $8.57 \%$ \\
\cline { 2 - 6 } & Class 3 & $5.45 \%$ & $3.64 \%$ & $\mathbf{8 4 . 5 5 \%}$ & $6.36 \%$ \\
\cline { 2 - 6 } & Class 4 & $1.81 \%$ & $2.73 \%$ & $3.64 \%$ & $\mathbf{9 1 . 8 2 \%}$ \\
\hline
\end{tabular}

Classic DTW ( $c=0$ ) scored the highest classification accuracy (91.97\%) for the collected dataset of 500 live MBPs. Table 4 shows the comparative statistics related to the classification accuracy for the studied compression rates. The results demonstrate that the accuracy of recognition of the MBPs' PAA is inversely proportional to $c$, i.e. the highest compression rates produced the least classification accuracies. This was also emphasized through the calculation of the standard mean error, which increased with the increasing compression values.

Table 4 PDTW classification accuracies

\begin{tabular}{lccccc}
\hline & $\begin{array}{c}\text { Classic } \\
\text { DTW }(\boldsymbol{c = 0})\end{array}$ & $\boldsymbol{c}=\mathbf{2 . 0 0}$ & $\boldsymbol{c}=\mathbf{4 . 0 0}$ & $\boldsymbol{c}=\mathbf{5 . 0 0}$ & $\boldsymbol{c}=\mathbf{8 . 0 0}$ \\
\hline Arithmetic mean (\%) & 91.97 & 91.95 & 91.05 & 90.91 & 90.00 \\
\hline Median (\%) & 95.00 & 95.00 & 95.00 & 95.00 & 95.00 \\
\hline Standard deviation & 10.89 & 10.92 & 12.66 & 12.78 & 13.09 \\
\hline Relative standard deviation $(\boldsymbol{\%})$ & 11.85 & 11.88 & 13.90 & 14.06 & 14.55 \\
\hline Standard error of the mean & 2.32 & 2.33 & 2.70 & 2.73 & 2.79 \\
\hline
\end{tabular}


The accuracies are visualized in the Box and Whisker plots in Figure 8, with classic DTW symbolized with a compression rate of $c=0$. As $c$ increases, more outliers were recorded. The obtained values also show the expansion of the lower minimum bound with the increasing value of $c$, signifying that the degree of accuracy deteriorates as MBPs become more compressed.

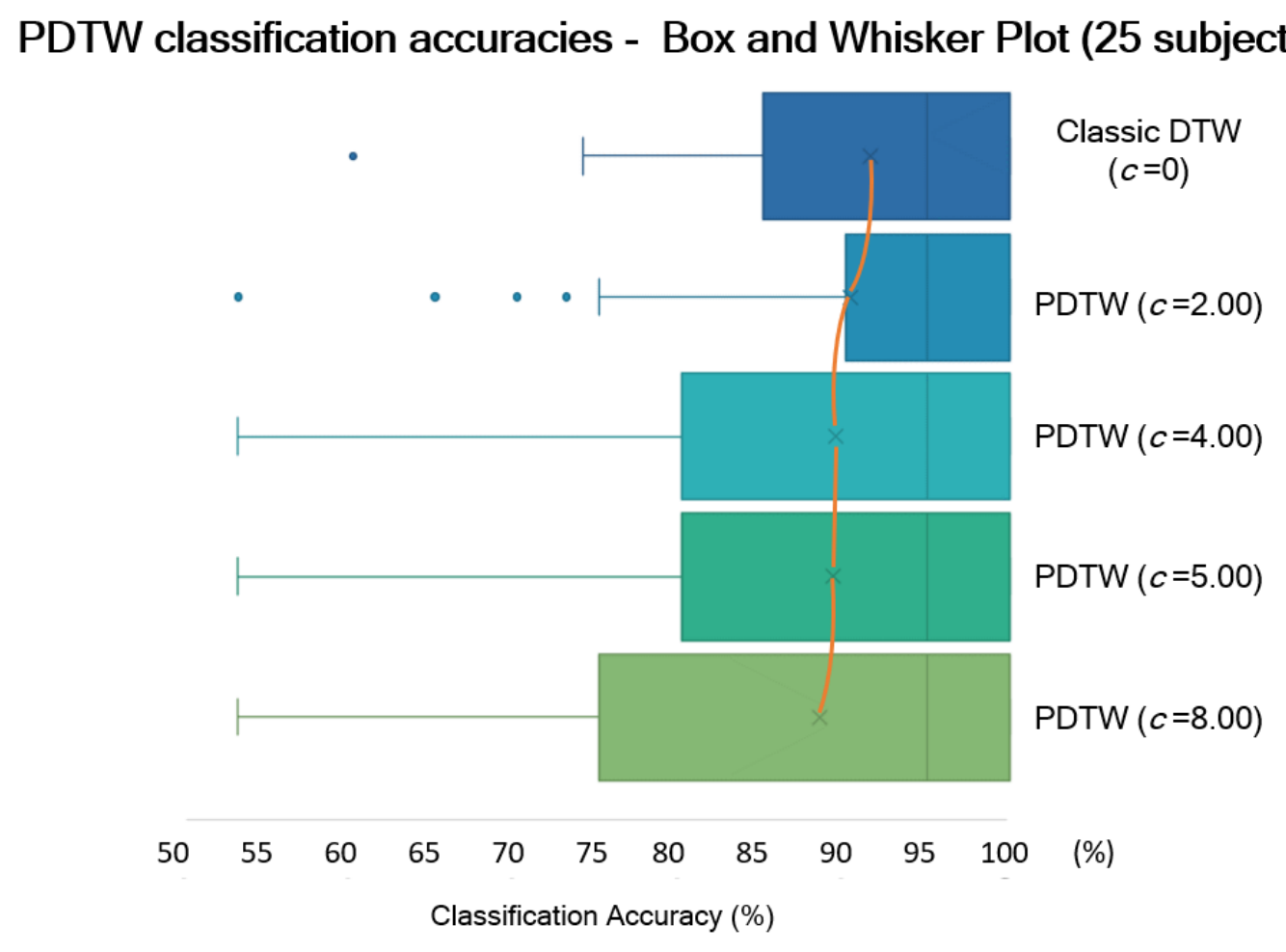

Figure 8 Analysis of PDTW's classification accuracies via a Box and Whisker plot showing the classification accuracy decreasing with the increasing compression rate.

The results demonstrate a tradeoff between the speed of the classified MBP series and the accuracy of the classification. As shown in Figure 9, the results obtained using a PC with 16 GB of RAM capacity showed a significant reduction in the time requirements as the MBP sequences became more compressed. MATLAB was used to obtain these values by measuring the classification time it takes for each subject's 20 live MBPs to be classified against their 40 training MBPs. The results were aggregated, and the average was computed. 


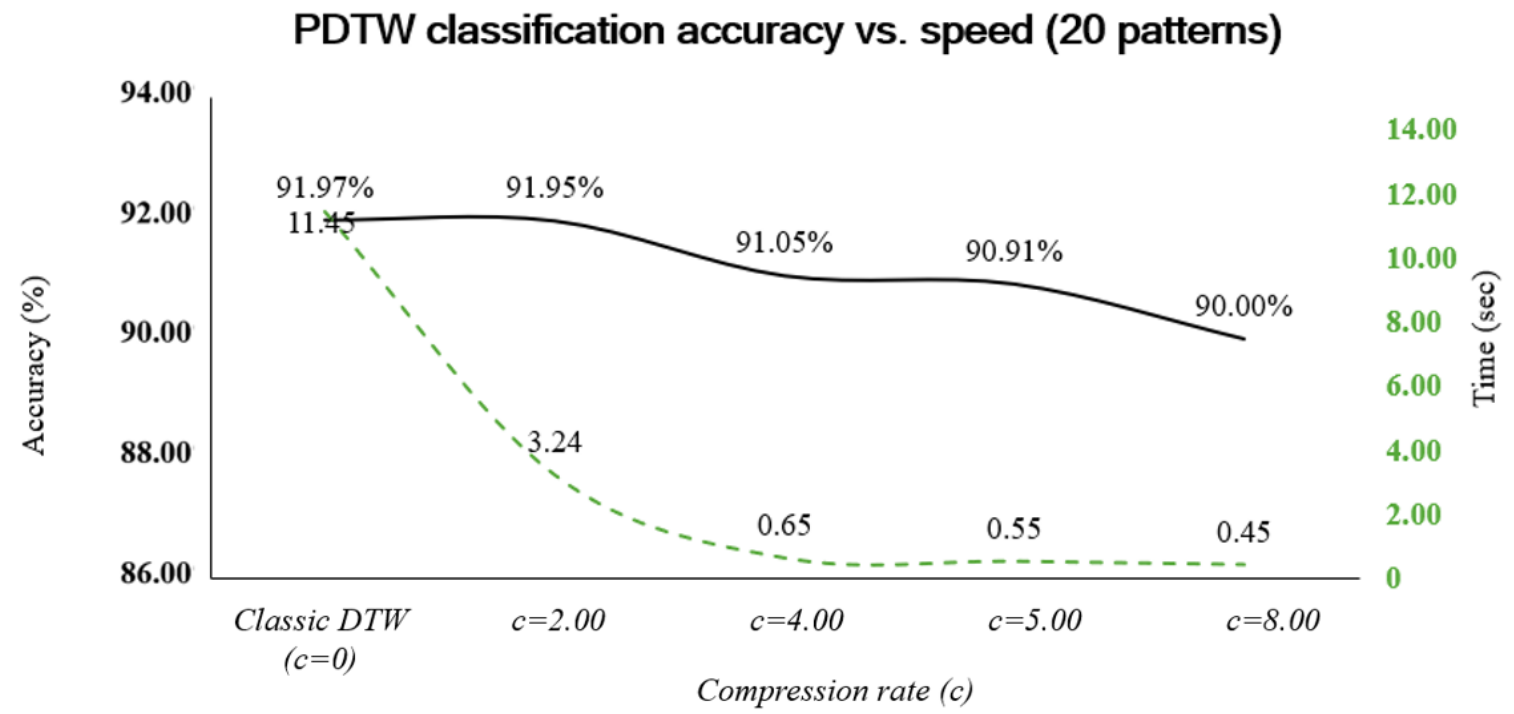

RAM Capacity:

16 GB $\quad$ Average Accuracy (\%) - - - Average Time (sec)

Figure 9 PDTW classification accuracy vs. classification time

As the algorithm searches a subject's 40 training MBPs to classify a live MBP, the obtained classification accuracy figures were very similar for the uncompressed DTW results and those with the lower compression rates, e.g. $c=2.00$, with a significant drop of 8.21 seconds $(\mathrm{sec})$ in this case. The time complexity (and in turn the demand on the memory requirements) continues to decrease with the increasing $c$ value. However, the reduction comes at the cost of a higher standard mean of error, as shown in Table 4.

\section{Discussion}

The acquisition of the collimated MBPs using the DAPDS's low pressure sensor connected to a diffuser cap aided in the detection of a comprehensive signal, comprising both the positive (exhaling) and negative (inhaling) components representative of the subject's selected breathing variation. The previous study [5] in this area utilized the acoustic detection of breathing patterns, which were typically represented by an all-positive waveform. However, the magnitudes of the detected signal components, especially the incoming inhalation signals, did not impose enough pressure on the microphone's diaphragm to generate a comprehensive recording. This was addressed in the current study through the usage of the DAPDS hardware system, including a bidirectional pressure sensing unit. The pressure sensing DAPDS system comprehensively detects the inhalations and exhalations with an equal weight on the positive and negative profiles of breathing and minimal background variations. The obtained figures demonstrate that the usage of the DAPDS along with an elastic, DTW measure could improve the overall performance of the platform. This is primarily attributed to the ability of the DAPDS to represent the detected MBPs in a more comprehensive format. 
The collected MBP sets demonstrate that younger subjects (average age: 18-23) tend to select MBPs containing a higher frequency content. The quality of all recorded MBP depends on the orientation and position of the diffuser cap with respect to the mouth, and on the related configuration of the air tube. However, such dependability was minimal and had minor effects on the results of the live and training datasets collected from the same subject. The increased familiarity of the subjects with the MBPI platform was seen to continue to positively impact the accuracy of the supervised ML classification, and the subjects' ability to use the platform ameliorated as the study progressed. This was emphasized as the last recorded class (class 4) for all subjects showed the least number of errors with regards to the inability to repeat an MBP or to omit it.

The examination of PDTW's performance to manage the computational complexity of the classical DTW, shown in the constructed Box and Whisker plots (Figure 8), resulted in an acceptable trade-off by recording a classification accuracy of $91.05 \%$ at $0.65 \mathrm{sec}$ for PDTW $c=4.00$ (RAM: $16 \mathrm{~GB}$ ) in comparison to $91.97 \%$ at $11.45 \mathrm{sec}$ for classical DTW ( $c=0)$. Such a time requirement reduction could aid in terms of AAC practicality, especially as the DAPDS progresses towards future portable and miniaturized configurations. The improvement in memory access time and classification signifies that running the AAC platform application on a device with a lower RAM capacity (e.g. 4 GB, or 8 GB) could be managed. This will ensure that a wide range of AAC users could use the device swiftly, as the platform progresses over its next generation. This is also beneficial in improving the system's present AAC speed restrictions to allow for more narrative-like, realistic voice outputs, or the ability to join in conversations. Table 5 highlights the differences between the main elements of this study, and our previous work in [5].

Table 5 Highlighting the differences between this study and the work in [5], and the evolution towards the DAPDS.

\begin{tabular}{|c|c|c|}
\hline & $\begin{array}{l}\text { MBP Interpretation using the } \\
\text { DAPDS }\end{array}$ & $\begin{array}{l}\text { MBP Interpretation using Acoustic } \\
\text { Microphone Recordings in [5] }\end{array}$ \\
\hline Aims & $\begin{array}{l}\text { Establishment and validation } \\
\text { of the DAPDS platform to } \\
\text { comprehensively detect } \\
\text { MBPs and Enhancement of } \\
\text { the speed of output } \\
\text { generation to produce the } \\
\text { spoken output with } \\
\text { minimum delay. }\end{array}$ & $\begin{array}{l}\text { Establishment of the MBPI } \\
\text { system architecture to translate } \\
\text { MBPs into spoken words via a } \\
\text { lost-cost unidirectional } \\
\text { microphone. }\end{array}$ \\
\hline $\begin{array}{l}\text { Collected } \\
\text { Datasets }\end{array}$ & $\begin{array}{l}\text { Signals: Pressure-time } \\
\text { readings. } \\
\text { Dataset of } 1500 \text { MBPs } \\
\text { collected from } 25 \text { healthy } \\
\text { participants with no history } \\
\text { of respiratory illnesses. }\end{array}$ & $\begin{array}{l}\text { - Signals: Acoustic recordings. } \\
\text { - Dataset of } 1380 \text { MBPs collected } \\
\text { from } 23 \text { healthy participants with } \\
\text { no history of respiratory } \\
\text { illnesses. }\end{array}$ \\
\hline Hardware & $\begin{array}{l}\text { Low differential pressure } \\
\text { sensor configured with a } \\
\text { microcontroller board. }\end{array}$ & $\begin{array}{l}\text { - Back-electret headband } \\
\text { microphone interfaced with a } \\
\text { PC. }\end{array}$ \\
\hline
\end{tabular}




\begin{tabular}{|c|c|c|}
\hline Methods & $\begin{array}{l}\text { Establishment of the DAPDS } \\
\text { platform for the } \\
\text { comprehensive collection of } \\
\text { MBPs. } \\
\text { Application of PDTW } \\
\text { processing of acquired } \\
\text { dataset for a faster } \\
\text { production of the spoken } \\
\text { words and testing the trade- } \\
\text { off of accuracy and speed. } \\
\text { - Validation of the developed } \\
\text { prototype system via the } \\
\text { presented experimental } \\
\text { protocol. }\end{array}$ & $\begin{array}{l}\text { Interfacing a low-cost acoustic } \\
\text { transducer (microphone) with a } \\
\text { PC and pre-processing the } \\
\text { signals for noise removal. } \\
\text { Application of classic DTW to } \\
\text { test the capabilities of using } \\
\text { dynamic programming for the } \\
\text { classification of acoustic MBPs. } \\
\text { Validation of the developed } \\
\text { prototype system via an } \\
\text { experimental protocol. }\end{array}$ \\
\hline Results & $\begin{array}{l}\text { Usage of the pressure sensor } \\
\text { increases the sensitivity of } \\
\text { the overall platform to } \\
\text { comprehensively detect } \\
\text { MBPs. } \\
\text { Overall classification } \\
\text { accuracy of } 91.05 \% \text { for the } \\
\text { selected patterns using } \\
\text { PDTW with a compression } \\
\text { factor of } c=4.00 \text {. } \\
\text { Faster speed of production of } \\
\text { the spoken output using the } \\
\text { tested PDTW algorithm in } \\
\text { comparison with classic } \\
\text { DTW. }\end{array}$ & $\begin{array}{l}\text { - Usage of the acoustic transducer } \\
\text { (microphone) restricted the } \\
\text { information content of the } \\
\text { signals; however, the results } \\
\text { demonstrated a systematic } \\
\text { reliability of } 89.00 \% \text { with } \\
\text { increased user-familiarity with } \\
\text { the platform. } \\
\text { - Overall MBP classification } \\
\text { accuracy of } 86.00 \% \text { using classic } \\
\text { DTW. }\end{array}$ \\
\hline
\end{tabular}

As an intended AAC platform, the current study tests the usage of four distinct MBPs for the generation of a spoken output based on a user-defined library. Although the MBPs are customizable, expanding the library to include further contexts in which the recognition is possible would be explored in the forthcoming studies. This will enable the usage of the same MBPs in different user contexts to encode distinct messages. A bidirectional learning process is envisioned, where the user and the system would continue to learn from each other to adapt the DAPDS platform to each user's individual needs. The recommendation is for the DAPDS to be tested with the help of individuals with speech disabilities in the near future. A suitable means of selecting associated phrases and sentences for specific MBPs for speech disabled subjects would also need investigating. Existing AAC systems used in conjunction with the DAPDS could be used to aid this task or sets of phrases provided to the user would be available for selection. The appropriate method would depend on the type of speech disability.

\section{Conclusion}


The translation of MBPs into SMSW is presented in this study. The development of the DAPDS, including the airflow sensing mechanism, supervised ML algorithm, and the breath sensing assumptions are described. The customization of the solution to accept MBPs that are user-selected has been emphasized. This enables each user to adapt the system to their individual needs, including a degree of freedom in the formation of MBPs and the selection of the associated phrases and sentences. The training set creation and the dataflow to produce SMSW via classical 1-NN PDTW classifiers are detailed. The processing speed was tested at different resolutions of the PDTW algorithm to identify the point of balance between the accuracy of the system and the memory requirements for real-time synthesized speech production.

The system has been tested for the recognition of four distinct patterns, which could be utilized to mean different messages in different user contexts. The MBPs classification accuracies of the system were obtained by aggregating the results of 25 subjects $(\mathrm{N}=25)$, generating an array of 1500 MBPs. An overall empirical systematic reliability of over $91 \%$ for supervised ML using PDTW $(c=4.00)$ with a robust reduction in the memory requirements was recorded. The outcomes from the present system contribute to the detection and recognition of a comprehensive profile of breathing, both during the speaking and nonspeaking human phases. Dynamic airflow sensing solutions in combination with deep learning (DL) of breathing signatures can be recommended for improved breath activated AAC applications and the exploration of breath biometric decoding.

\section{Acknowledgements}

The authors would like to acknowledge the support of First Sensor for the provision of the low differential pressure sensor, as well as the support of Loughborough University in the conduction of this research.

\section{Author Contributions}

Y.E. carried out the study, the data processing, and wrote the manuscript. S.H. structured the manuscript, analyzed the outcomes, and supervised the study. K.B.-M. jointly supervised the study and reviewed the manuscript. D.K. provided background knowledge and advised on original work, co-supervised the study. W.W. and P.H. edited components related to AAC updating terms and language, and indicated the forthcoming AAC prospect. A.G. and V.K. discussed AAC clinical application concept with an identified procedure in the coming phase. 


\section{References}

1. Light, J.; McNaughton, D.; Beukelman, D.; Fager, S.K.; Fried-Oken, M.; Jakobs, T.; Jakobs, E. Challenges and opportunities in augmentative and alternative communication: Research and technology development to enhance communication and participation for individuals with complex communication needs. AAC Augment. Altern. Commun. 2019, 35, 1-12. https://doi.org/10.1080/07434618.2018.1556732

2. Wilkinson, K.M.; Mitchell, T. Eye Tracking Research to Answer Questions about Augmentative and Alternative Communication Assessment and Intervention. Augment. Altern. Commun. 2014, 30, 106-119. https://doi.org/10.3109/07434618.2014.904435

3. Gibbons, C.; Beneteau, E. Functional Performance Using Eye Control and Single Switch Scanning by People With ALS. Perspect. Augment. Altern. Commun. 2010, 19, 64-69. https://doi.org/10.1044/aac19.3.64

4. Chaudhary, U.; Birbaumer, N.; Curado, M.R. Brain-Machine Interface (BMI) in paralysis. Ann. Phys. Rehabil. Med. 2015, 58, 9-13.

https://doi.org/10.1016/j.rehab.2014.11.002

5. Elsahar, Y.; Bouazza-Marouf, K.; Kerr, D.; Gaur, A.; Kaushik, V.; Hu, S. Breathing Pattern Interpretation as an Alternative and Effective Voice Communication Solution. Biosensors 2018, 8, 48. https://doi.org/10.3390/bios8020048

6. Fager, S.; Bardach, L.; Russell, S.; Higginbotham, J. Access to augmentative and alternative communication: New technologies and clinical decision-making. J. Pediatr. Rehabil. Med. 2012, 5, 53-61. https://doi.org/10.3233/PRM-2012-0196

7. Elsahar, Y.; Hu, S.; Bouazza-Marouf, K.; Kerr, D.; Mansor, A. Augmentative and Alternative Communication (AAC) Advances: A Review of Configurations for Individuals with a Speech Disability. Sensors 2019, 19, 1911. https://doi.org/10.3390/s19081911

8. Glennen, S.L. Augmentative and alternative communication systems. In The Handbook of Augmentative and Alternative Communication; Cengage Learning: Boston, MA, USA, 1997; pp. 59-69. ISBN 9780323096317.

9. Light, J.; McNaughton, D. The Changing Face of Augmentative and Alternative Communication: Past, Present, and Future Challenges. Augment. Altern. Commun. 2012, 28, 197-204. https://doi.org/10.3109/07434618.2012.737024

10. Black, R.; Waller, A.; Tintarev, N.; Reiter, E.; Reddington, J. A mobile phone based personal narrative system. In Proc. of the 13th international ACM SIGACCESS conference on Computers and accessibility - ASSETS '11; ACM Press, New York, USA, 2011; p. 171. https://doi.org/10.1145/2049536.2049568

11. Dempster, M.; Alm, N.; Reiter, E. Automatic generation of conversational utterances and narrative for augmentative and alternative communication: a prototype system. In Proc. NAACL HLT 2010 Workshop Speech Language Processing Assistive Tech.; Association for Computational Linguistics: Los Angeles, California, 2010; pp. 10-18.

12. Kerr, D.; Bouazza-Marouf, K.; Gaur, A.; Sutton, A.; Green, R. A breath controlled AAC system. In Proc. CM2016 Natl. AAC Conf, Apr 2016; pp. 11-13. 
13. Waller, A. Telling tales: Unlocking the potential of AAC technologies. Int. J. Lang. Commun. Disord. 2019, 1-11. https://doi.org/10.1111/1460-6984.12449

14. Nijboer, F.; Plass-Oude Bos, D.; Blokland, Y.; van Wijk, R.; Farquhar, J. Design requirements and potential target users for brain-computer interfacesrecommendations from rehabilitation professionals. Brain-Computer Interfaces 2014, 1, 50-61. https://doi.org/10.1080/2326263X.2013.877210

15. Son, S.-H.; Sigafoos, J.; O’Reilly, M.; Lancioni, G.E. Comparing two types of augmentative and alternative communication systems for children with autism. Pediatr. Rehabil. 2006, 9, 389-395. https://doi.org/10.1080/13638490500519984

16. Reichle, J.; Simacek, J.; Wattanawongwan, S.; Ganz, J. Implementing Aided Augmentative Communication Systems with Persons Having Complex Communicative Needs. Behav. Modif. 2019, 43, 841-878. https://doi.org/10.1177/0145445519858272

17. Shorrock, T.; MacKay, D.; Ball, C. Efficient Communication by Breathing. In Deterministic and Statistical Methods in Machine Learning; Springer: Heidelberg/Berlin, Germany, 2005; pp. 88-97. https://doi.org/10.1007/11559887 5

18. Plotkin, A.; Sela, L.; Weissbrod, A.; Kahana, R.; Haviv, L.; Yeshurun, Y.; Soroker, N.; Sobel, N. Sniffing enables communication and environmental control for the severely disabled. In Proc. Natl. Acad. Sci. USA 2010, 107, 14413-14418. https://doi.org/10.1073/pnas.1006746107

19. Garcia, R.G.; Ibarra, J.B.G.; Paglinawan, C.C.; Paglinawan, A.C.; Valiente, L.; Sejera, M.M.; Bernal, M.V.; Cortinas, W.J.; Dave, J.M.; Villegas, M.C. Wearable augmentative and alternative communication device for paralysis victims using Brute Force Algorithm for pattern recognition. In Proc. 2017 IEEE 9th Int'l Conf Humanoid, Nanotech, Info Tech, Commun. Control, Environ. Manag. (HNICEM), Manila, Philippines, Dec 2017; pp. 1-6. https://doi.org/10.1109/HNICEM.2017.8269554

20. Simion, E. Augmentative and Alternative Communication-Support for People with Severe Speech Disorders. Procedia-Soc. Behav. Sci. 2014, 128, 77-81. https://doi.org/10.1016/j.sbspro.2014.03.121

21. Benedikt, L.; Kajic, V.; Cosker, D.; Rosin, P.L.; Marshall, D. Facial Dynamics in Biometric Identification. In Proc. British Machine Conf. BMVA Press; Leeds, UK, Sept. 2008; pp. 107.1-107.10. https://doi.org/10.5244/c.22.107

22. Mondal, T.; Ragot, N.; Ramel, J.Y.; Pal, U. Performance evaluation of DTW and its variants for word spotting in degraded documents. In Proc. Int'l Conf. Doc. Analysis and Recog, ICDAR; 2015; Vol. 2015-Novem, pp. 1141-1145. https://doi.org/10.1109/ICDAR.2015.7333939

23. Keogh, E.J.; Pazzani, M.J. Scaling up dynamic time warping for datamining applications. In Proc. of the 6th ACM SIGKDD Int'l Conf Knowledge Discovery Data Mining; 2004, 285-289. https://doi.org/10.1145/347090.347153 


\title{
Authors Contributions
}

\section{A Study of Decodable Breathing Patterns for Augmentative and Alternative Communication}

\author{
Yasmin Elsahar ${ }^{1}$, Sijung Hu ${ }^{1 *}$, Kaddour Bouazza-Marouf ${ }^{1}$, David Kerr ${ }^{1}$, Will Wade ${ }^{2}$, Paul \\ Hewett $^{2}$, Atul Gaur ${ }^{3}$, and Vipul Kaushik ${ }^{3}$ \\ ${ }^{1}$ Wolfson School of Mechanical, Electrical, and Manufacturing Engineering, Loughborough \\ University, Loughborough, LE11 3TU, UK \\ ${ }^{2}$ ACE Centre, Hollinwood Business Centre, Albert Street, Oldham, OL8 3QL,UK \\ ${ }^{3}$ University Hospitals of Leicester NHS Trust, Leicester, LE3 9QP, UK \\ *Correspondence: S.Hu@lboro.ac.uk
}

\section{Detailed Authors Contributions}

Y.E. carried out the study, the data processing, and wrote the manuscript.

S.H. structured the manuscript, analyzed the outcomes, and supervised the study.

K.B.-M. jointly supervised the study and reviewed the manuscript.

D.K. provided background knowledge and advised on original work, co-supervised the study.

W.W. and P.H. edited components related to AAC updating terms and language, and indicated the forthcoming AAC prospect.

A.G. and V.K. discussed AAC clinical application concept with an identified procedure in the coming phase. 


\section{${ }^{*}$ Declaration of Interest Statement}

\section{Declaration of interests}

$\bigotimes$ The authors declare that they have no known competing financial interests or personal relationships that could have appeared to influence the work reported in this paper.

$\square$ The authors declare the following financial interests/personal relationships which may be considered as potential competing interests:

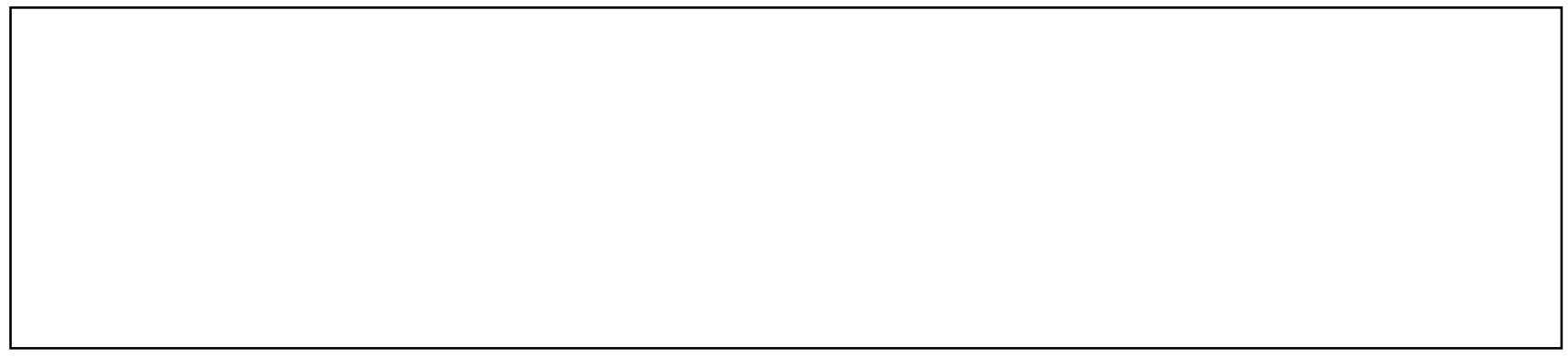

